\title{
Tracking of Acceleration with HNJ Method
}

\author{
A. Ruggiero
}

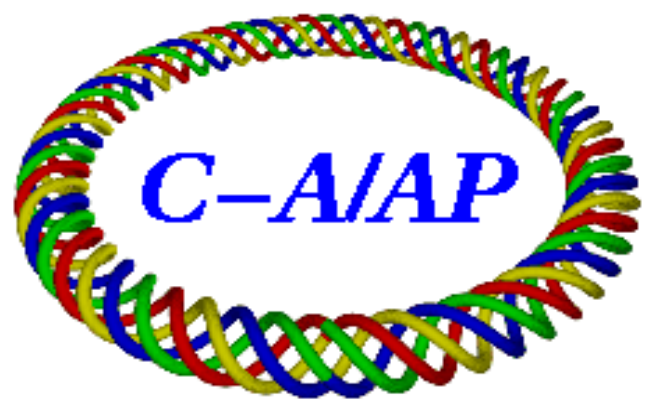

Collider-Accelerator Department Brookhaven National Laboratory Upton, NY 11973

Notice: This document has been authorized by employees of Brookhaven Science Associates, LLC under Contract No. DE-AC02-98CH10886 with the U.S. Department of Energy. The United States Government retains a nonexclusive, paid-up, irrevocable, world-wide license to publish or reproduce the published form of this document, or allow others to do so, for United States Government purposes. 


\title{
Tracking of Acceleration with HNJ Method
}

\author{
Alessandro G. Ruggiero \\ Brookhaven National Laboratory, PO Box 5000, Upton, NY 11973, USA
}

\begin{abstract}
After reviewing the principle of operation of acceleration with the method of Harmonic Number Jump (HNJ) in a Fixed-Field Alternating-Gradient (FFAG) accelerator for protons and heavy ions, we report in this talk the results of computer simulations performed to assess the capability and the limits of the method in a variety of practical situations. Though the study is not yet completed, and there still remain other cases to be investigated, nonetheless the tracking results so far obtained are very encouraging, and confirm the validity of the method.
\end{abstract}

Keywords: Fixed-Field Alternating-Gradient Accelerator; Tracking; RF Acceleration; Harmonic Number Jump. PACS: $29.27 . \mathrm{Bd}$

\section{THE METHOD OF ACCELERATION BY HNJ}

Let us start by describing the particle beam and the accelerator where acceleration by HNJ is performed [1-3]. The FFAG accelerator has a circumference $\mathrm{C}=\mathrm{C}(w)$ that is a function of the particle kinetic energy $w$. At one location $\mathrm{O}$ there is a single RF cavity system operating at constant frequency $f_{\mathrm{RF}}$. The beam is made of a number $\mathrm{M}$ of bunches that we assume all alike and point-like, equally spaced from each other and all at the same kinetic energy $w$. There is a gap in the beam of sufficiently long extension as shown in Figure 1. The beam bunches have also the same number $\mathrm{N}$ of particles. At a certain time $t_{\mathrm{n}}$ that may correspond to the situation of Figure 1 , that is during the $\mathrm{n}$ th revolution, all bunches have traversed the RF cavity, and have now the kinetic energy $w_{\mathrm{n}}$ and revolution frequency $f_{\mathrm{n}}$. Parameters are adjusted so that $f_{\mathrm{RF}}$ and $f_{\mathrm{n}}$ are in an integral relationship $f_{\mathrm{RF}}=h_{\mathrm{n}} f_{\mathrm{n}}$ with $h_{\mathrm{n}}$ an integer, the harmonic number during the $\mathrm{n}$-th revolution. At all times the number $\mathrm{M}$ of bunches is considerably lower than the harmonic number, that is $h_{\mathrm{n}}>\mathrm{M}$. As soon the last beam bunch has crossed the cavity, and before the arrival of the first bunch, the RF peak voltage and phase are re-adjusted respectively to $V_{n}$ and $\phi_{n}$, if so required, or, otherwise, kept unchanged. Then the full length of the beam pulse enters again the RF system, and all particles in the beam receive the same energy gain

$$
\mathrm{U}_{\mathrm{n}}=e(\mathrm{Q} / \mathrm{A}) \mathrm{V}_{\mathrm{n}} \sin \phi_{\mathrm{n}}
$$

where $e$ is the elementary electric charge, Q the ion charge state, and A the ion mass number. Particle energy and energy gain are given in atomic mass unit. After the full beam has gone through the RF system, the beam energy during the $(\mathrm{n}+1)$-th revolution is

$$
w_{\mathrm{n}+1}=w_{\mathrm{n}}+\mathrm{U}_{\mathrm{n}}
$$

The new revolution frequency is $f_{\mathrm{n}+1}$, and the new harmonic number

$$
h_{\mathrm{n}+1}=h_{\mathrm{n}}-\Delta_{\mathrm{n}}
$$

where $\Delta_{\mathrm{n}}$ is a positive integer number: the harmonic number jump that may also vary from turn to turn. We are intentionally assuming the case the beam energy is below the ring transition energy, that explains the decrease of the harmonic number as shown in Eq. (3). Once the whole beam has crossed the RF cavity the cycle repeats by re- 
adjusting the RF voltage and phase to new values $V_{n+1}$ and $\phi_{n+1}$, or otherwise kept constant. Again it is assumed that every revolution the time gap $\left(h_{\mathrm{n}}-\mathrm{M}\right) / f_{\mathrm{RF}}$ is long enough to allow any required change of the RF parameters. In case the RF para meters are kept unchanged during the course of the acceleration cycle, the duration of the beam gap is of no direct consequence, except that no matter how small it should always be positive.

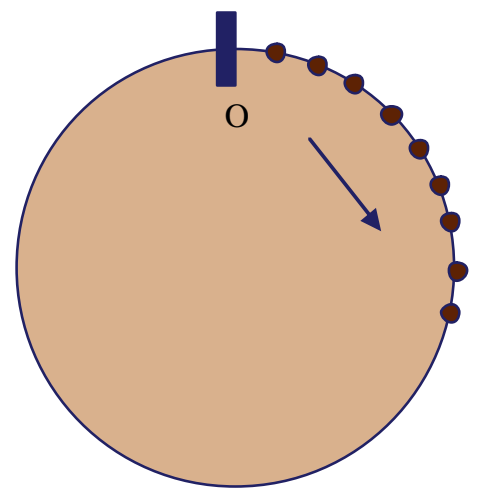

FIGURE 1. Beam Bunches circulating past an RF Cavity System in a FFAG Ring.

The cavity is placed at the location $\mathrm{O}$ where the ring lattice has dispersion (probably non-linear). Since the bunches during any revolution have the same energy $w_{\mathrm{n}}$, they all cross the cavity at the same radial position $x_{\mathrm{n}}$ that is a function, because of dispersion, of the kinetic energy $w_{\mathrm{n}}$. Quite generally, the RF voltage varies across the radial extension of the cavity so that

$$
\mathrm{V}_{\mathrm{n}}=\mathrm{V}\left(x_{\mathrm{n}}\right)=\mathrm{V}\left[x_{\mathrm{n}}\left(w_{\mathrm{n}}\right)\right]=\mathrm{V}\left(w_{\mathrm{n}}\right)
$$

There may be of course other mixed situations, with the RF voltage being also partially adjusted at every revolution. During the acceleration it is important that the energy gain Eq. (1) is programmed in such a way that the harmonic number jump $\Delta_{\mathrm{n}}$ has the required integer value. The revolution frequency

$$
f_{\mathrm{n}}=\beta_{\mathrm{n}} \mathrm{c} / \mathrm{C}_{\mathrm{n}}
$$

where $\beta_{\mathrm{n}} \mathrm{c}$ is the beam velocity that varies with the beam energy $w_{\mathrm{n}}$, and $\mathrm{C}_{\mathrm{n}}=\mathrm{C}\left(w_{\mathrm{n}}\right)$ the ring circumference during the $\mathrm{n}$-th revolution, is also in principle a function of the energy $w_{\mathrm{n}}$. For a non-isochronous FFAG ring (scaling and non-scaling) operating below the transition energy, usually the variation of the ring circumference with the beam energy is weak (though of course it can be computed exactly), and could be ignored, if so desired, with respect to the change of the beam velocity with its energy. Thus most of the change of the revolution frequency $f_{\mathrm{n}}$ is given by the beam velocity $\beta_{n} c$ and only in a very minor part by $C_{n}$. Let us start by neglecting the variation of the circumference $\mathrm{C}_{\mathrm{n}}$ with the beam energy, that is $\mathrm{C}_{\mathrm{n}}=\mathrm{C}_{0}$, a constant (for instance the value at the injection energy). In this case, combining Eq.s (5) with $f_{\mathrm{RF}}=h_{\mathrm{n}} f_{\mathrm{n}}$ we obtain

$$
\beta_{\mathrm{n}} h_{\mathrm{n}}=\text { invariant }
$$

where the invariant is equal, for instance, to the initial value $\beta_{0} h_{0}$ at injection.

\section{GENERIC FFAG PROTON DRIVER}

Let us apply the considerations above to the FFAG Proton Driver (PD) $(\mathrm{Q}=\mathrm{A}=1)$ that has been studied in the past [4]. It is made of two rings: the first for accele ration from 50 to $250 \mathrm{MeV}$, and the second from $250 \mathrm{MeV}$ to 1 $\mathrm{GeV}$. Let us examine the second ring where we propose acceleration by HNJ with a constant RF frequency around $805 \mathrm{MHz}$. The circumference (at injection) $\mathrm{C}_{0}=204 \mathrm{~m}$. The initial values are $h_{0}=893$ and $\beta_{0}=0.6136$. We also take the harmonic number jump $\Delta_{\mathrm{n}}=1$ at every revolution. We are then in position to estimate from Eqs. (3 and 6), with a simple iteration, $\beta_{\mathrm{n}}$ and $h_{\mathrm{n}}$ at every revolution. The final energy of $1 \mathrm{GeV}$ is reached after $n=267$ revolutions (corresponding to $0.252 \mathrm{~ms}$ acceleration time) when $\beta_{\mathrm{n}}=0.8753$ and $h_{\mathrm{n}}=626$, as shown in Figures 2 and 3 . Figure 4 
is the plot of $w_{\mathrm{n}}$ every revolution, and Figure 5 that of $\mathrm{U}_{\mathrm{n}}$. The same Figure 5 plots also the RF peak voltage $\mathrm{V}_{\mathrm{n}}$ for acceleration of protons at constant RF phase angle $\phi_{n}=60^{\circ}$ according to Eq. (1). It is seen that the energy gain $U_{n}$ and the RF peak voltage $V_{n}$ increase at very large rate during the acceleration cycle. It is also possible, again according to Eq. (1), to keep the RF peak voltage constant during the acceleration at the value corresponding to the end of the cycle, and vary the RF phase angle $\phi_{n}$. The result is plotted in Figure 6 for the value $\phi_{n}=60^{\circ}$ at the end of acceleration. Figure 7 plots other parameters relevant to the acceleration cycle.

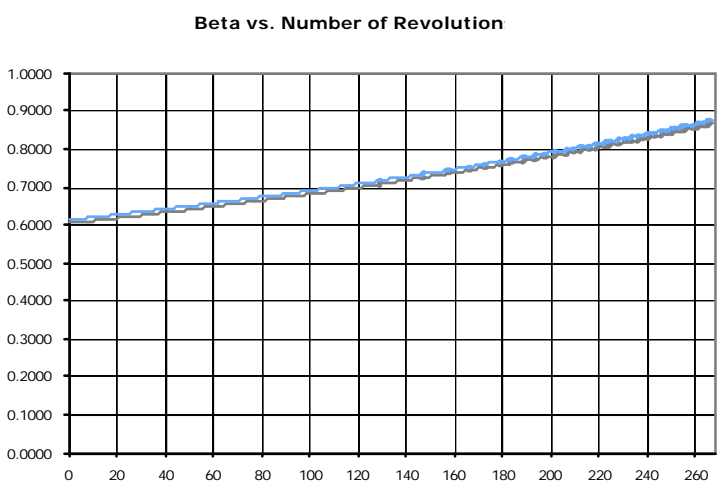

FIGURE 2. $\beta_{\mathrm{n}}$ for Proton Driver

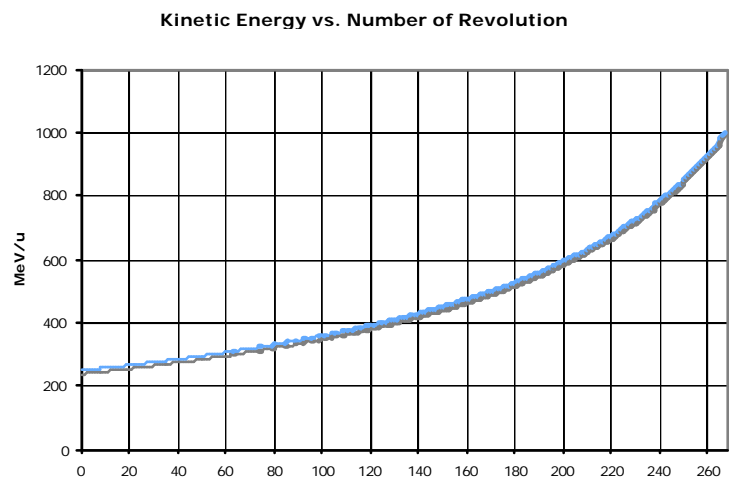

FIGURE 4. $w_{\mathrm{n}}$ for Proton Driver

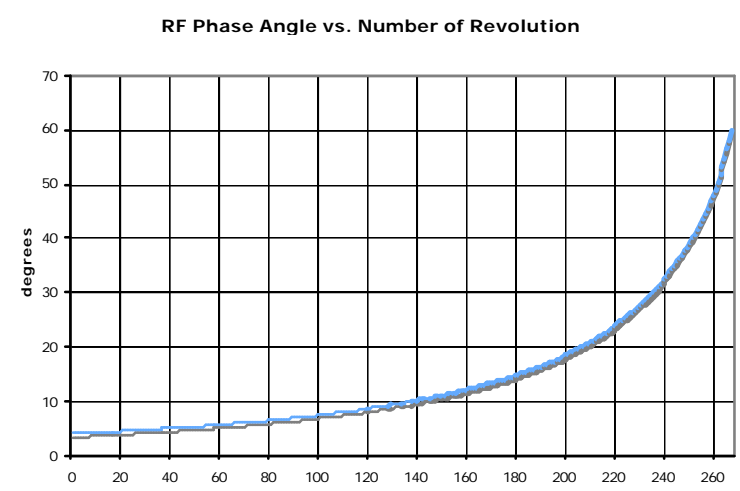

FIGURE 6. $\phi_{\mathrm{n}}$ for Proton Driver

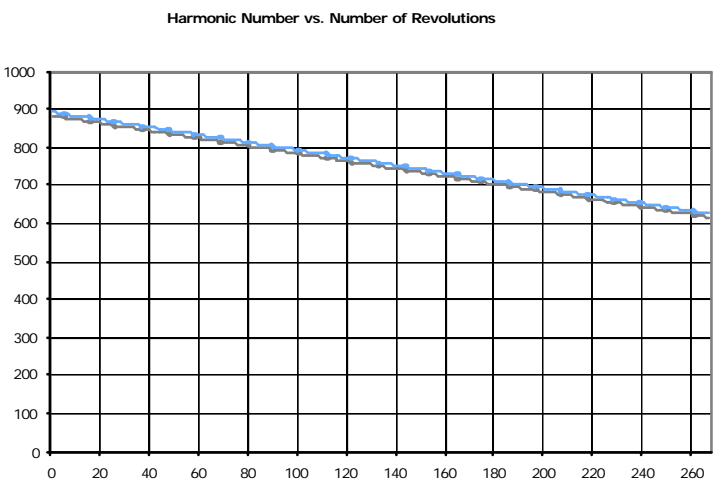

FIGURE 3. $h_{\mathrm{n}}$ for Proton Driver

Energy Gain, Peak Voltage vs. Number of Revolutio

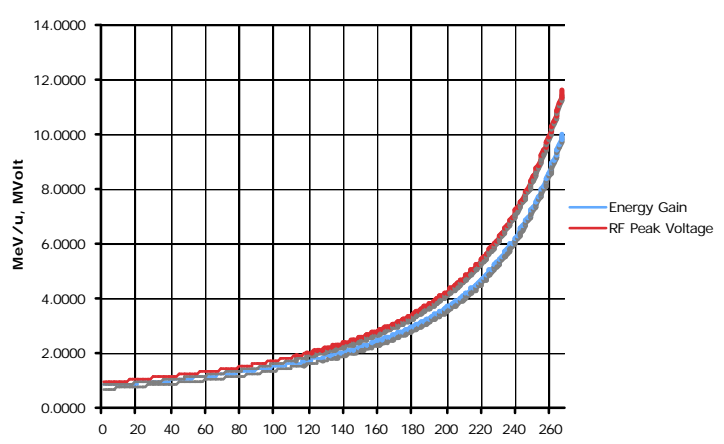

FIGURE 5. $U_{n}, V_{n}$ for Proton Driver

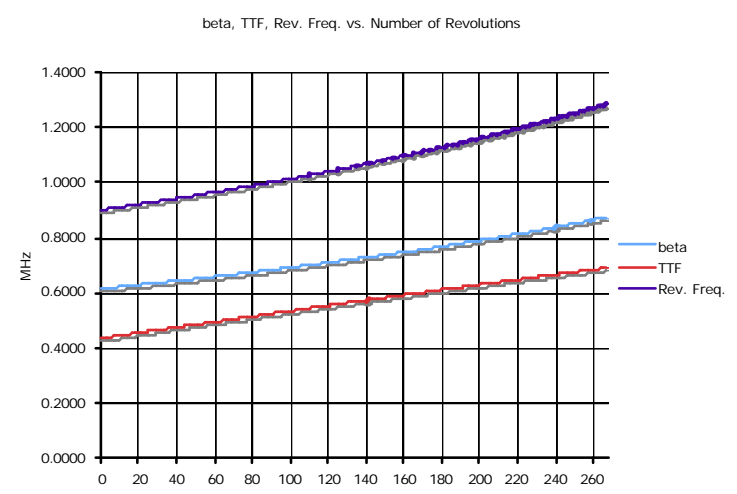

FIGURE 7. $\beta_{\mathrm{n}}$, TTF, $f_{\mathrm{n}}$ for Proton Driver 


\section{FFAG MAIN RING AT KURRI *}

We can repeat the same exercise for the FFAG main Ring at KURRI, Japan [5]. In this ring that also accelerates protons, the energy range is 20 to $150 \mathrm{MeV}$, and the circumference at injection $\mathrm{C}_{0}=28.5 \mathrm{~m}$. Though now the variation of the circumference with the beam energy is considerable larger when compared to the previous example (a relative variation of about $13 \%$ versus $0.3 \%$ ), we shall still assume that the circumference is about unchanged during acceleration so we can make use again of Eq. (6) with $\beta_{0}=0.2032$ and $h_{0}=234$. Considering the larger radial expansion of the beam trajectories, we take for this case a constant RF frequency around $f_{\mathrm{RF}}=500 \mathrm{MHz}$. The final energy value is reached after $n=148$ revolutions, corresponding to an acceleration period of $47.5 \mu \mathrm{s}$, when $\beta_{\mathrm{n}}=$ 0.533 and $h_{\mathrm{n}}=86$. The corresponding results, equivalent to those derived for the previous case, are displayed in Figures 8 to 13 .

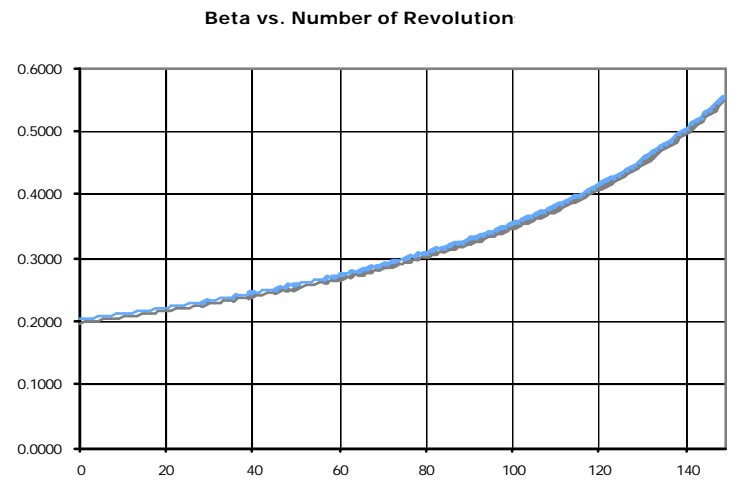

FIGURE 8. $\beta_{\mathrm{n}}$ for KURRI Main Ring

Kinetic Energy vs. Number of Revolution

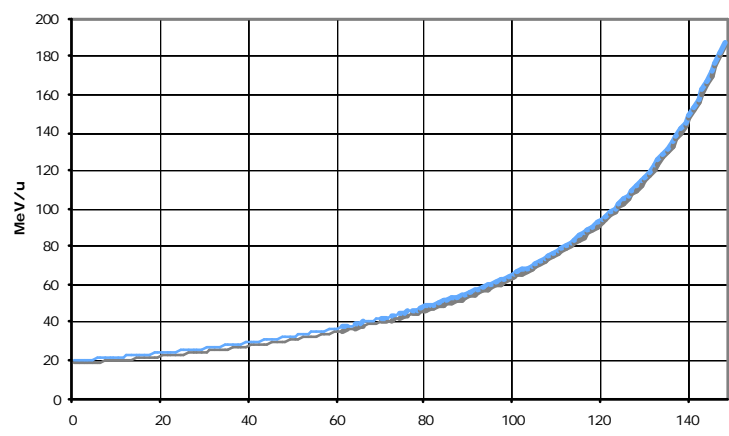

FIGURE 10. $w_{\mathrm{n}}$ for KURRI Main Ring

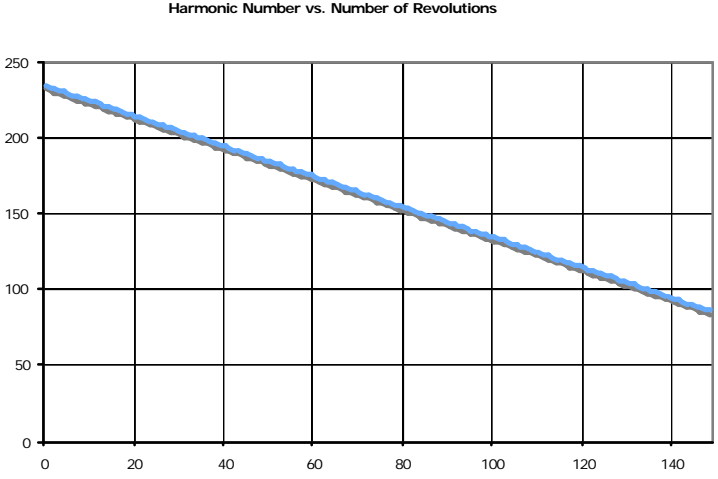

FIGURE 9. $h_{\mathrm{n}}$ for KURRI Main Ring

Energy Gain, Peak Voltage vs. Number of Revolutic

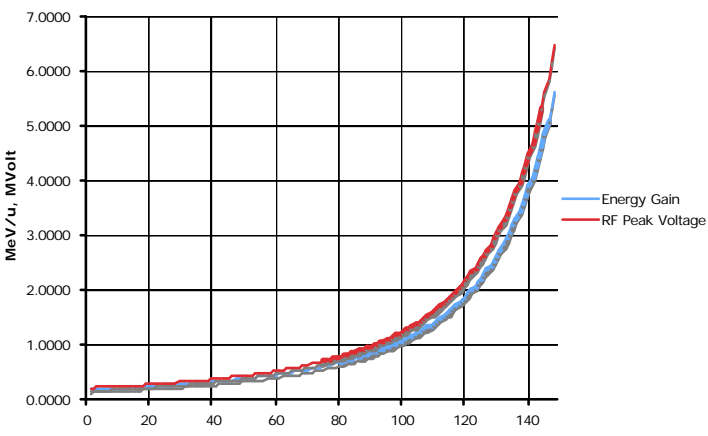

FIGURE 11. $U_{n}, V_{n}$ for KURRI Main Ring

\section{THE RF SYSTEM}

It is seen that in both cases considered the required energy gain per revolution increases at large rate during acceleration and reach exceedingly large values at the end of the acceleration cycle: $5.5 \mathrm{MeV} /$ turn in the KURRI ring, and $10 \mathrm{MeV} /$ turn for the PD ring. Such large energy gain can be obtained only with superconducting cavities that also allow at the same time high accelerating longitudinal field gradients. Single-cell cavities operating in $\mathrm{p}$ mode have a longitudinal gap $g=\beta_{0} \lambda / 2$ where $\beta_{0}$ is the reference beam velocity value to which all cavities are tuned, and $\lambda=\mathrm{c} / f_{\mathrm{RF}}$ is the RF wavelength. There is a Transit Time Factor (TTF), shown in Figures 7 and 13, that determines how much acceleration is provided by a cavity when it is traversed by a particle at velocity $\beta$ other than $\beta_{0}$, namely

\footnotetext{
* Kyoto University Research Reactor Institute
} 


$$
\operatorname{TTF}\left(\beta, \beta_{0}\right)=\sin \left(\mathrm{p} \beta_{0} / 2 \beta\right) /\left(\mathrm{p} \beta_{0} / 2 \beta\right)
$$

Rather than the RF peak voltage, it is the largest longitudinal axial field

$$
\xi_{\mathrm{n}}=\left(\mathrm{V}_{\mathrm{n}} / g\right) \operatorname{TTF}\left(\beta, \beta_{0}\right) \sin \phi_{\mathrm{n}}
$$

that can be achieved that defines the performance of superconducting cavities (typically a value not exceeding 20-30 MVolt $/ \mathrm{m}$ ). The following Table 1 summarizes the parameters for the two cases under consideration.

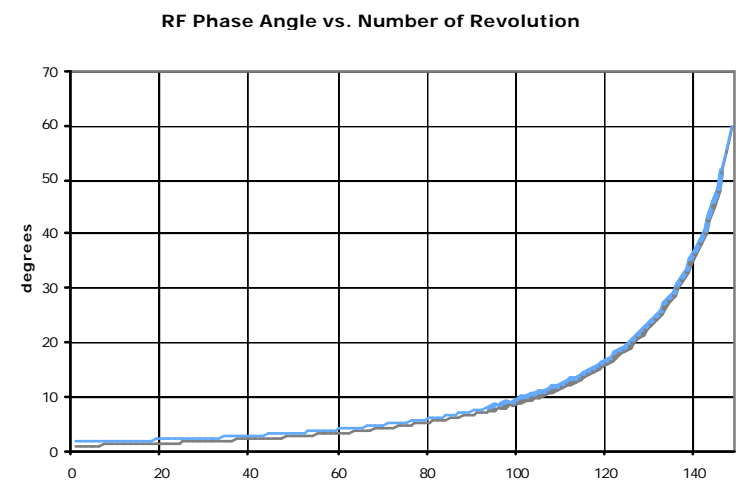

FIGURE 12. $\phi_{n}$ for KURRI Main Ring

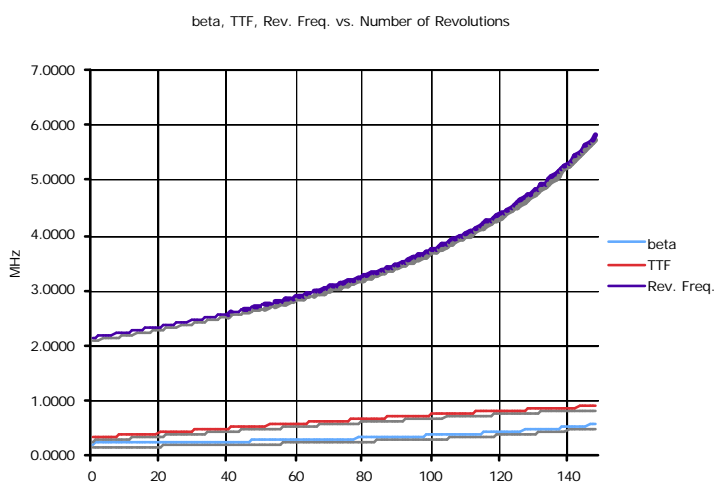

FIGURE 13. $\beta_{\mathrm{n}}$, TTF, $f_{\mathrm{n}}$ for KURRI Main Ring

TABLE 1. Parameters of the two RF Cavity Systems

\begin{tabular}{lcc}
\hline & KURRI Main Ring & Proton Driver \\
\hline$f_{\mathrm{RF}}, \mathrm{MHz}$ & 500.27 & 805.26 \\
$\lambda, \quad \mathrm{cm}$ & 59.93 & 37.23 \\
$\mathrm{~g}, \quad \mathrm{~cm}$ & 8.99 & 14.89 \\
$\beta_{0}$ & 0.30 & 0.80 \\
Number of Cavities & 4 & 6 \\
\hline
\end{tabular}

If a constant RF phase $\phi_{n}=60^{\circ}$ is maintained during operation, the longitudinal axial field $\xi_{\mathrm{n}}$ will have to vary as shown in Figures 14 and 15. To keep the maximum value of $\xi_{n}$ within acceptable limit, each RF station is made of a number of individually-powered, phase-independent single-cell cavities with parameters shown in Table 1. The largest axial field then is around $20 \mathrm{MVolt} / \mathrm{m}$ in both cases.

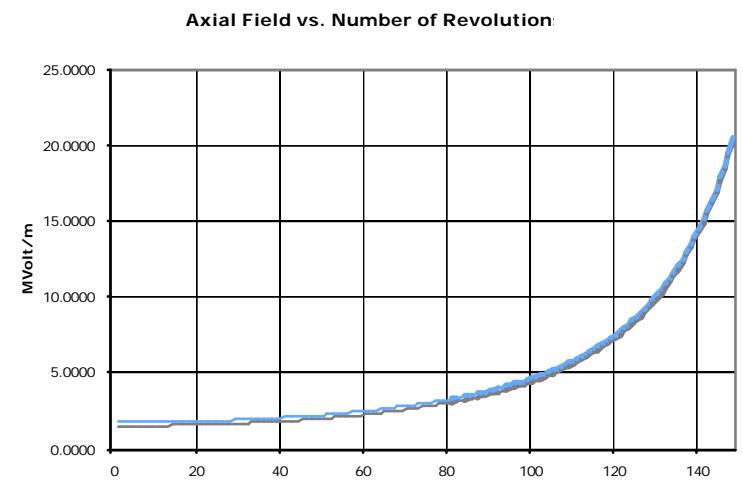

FIGURE 14. Axial Field for KURRI Main Ring

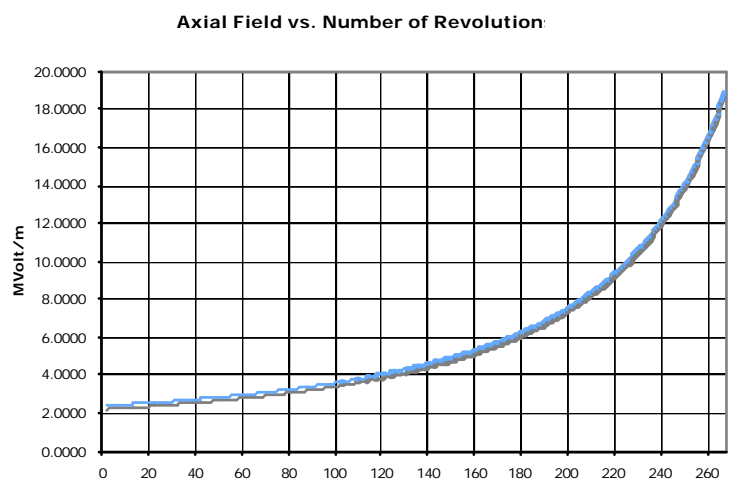

FIGURE 15. Axial Field for Proton Driver 


\section{MODES OF OPERATION}

There are two modes of operation:

(1) Constant RF peak voltage and varying RF phase angle. In this mode the cavity axial field is kept constant at the maximum value of $20 \mathrm{MVolt} / \mathrm{m}$, but the RF phase is varied as prescribed by Figures 6 and 12 for the required energy gain profile of Figures 5 and 11. Indeed the change of the RF phase angle is modest, at most a couple of degrees per revolution at the end of acceleration cycle, and should be possible to be executed by acting on the loading of the RF power system. Still it is needed to split the cavities in two groups of 2 cavities each, in the KURRI case, and in three groups also of two cavities in the PD case, because of the limited space of the long insertions (about $1 \mathrm{~m}$ for the PD case, and $0.4 \mathrm{~m}$ for the KURRI case). With this operation one loses the locality of the RF cavities that can only be partially recovered by placing the two or three groups in contiguous long drifts, and by paraphasing the cavities so that altogether they act as a single unit in one reference location in between.

(2) Constant RF Phase Voltage and RF axial Field that varies across the width of the cavities. We shall apply this to the Proton Driver case, though it applies as well also to the KURRI accelerator. Figure 16 displays the radial beam position across the cavity location at different energies. Figure 17 then plots the axial field versus radial position assuming constant RF phase. The results are now exact since they have been derived taking into account also the variation of the circumference with the beam energy. Also we took $3 \mathrm{RF}$ cavity systems located in contiguous long drift. Each system is made of three single cell cavities: the one in the middle in $\mathrm{TM}_{01}$ mode with an axial field of 16 MVolt $/ \mathrm{m}$ and the two on the sides in $\mathrm{TM}_{11}$ mode with axial field of $\pm 6 \mathrm{MVolt} / \mathrm{m}$ as shown in Figure 18 .
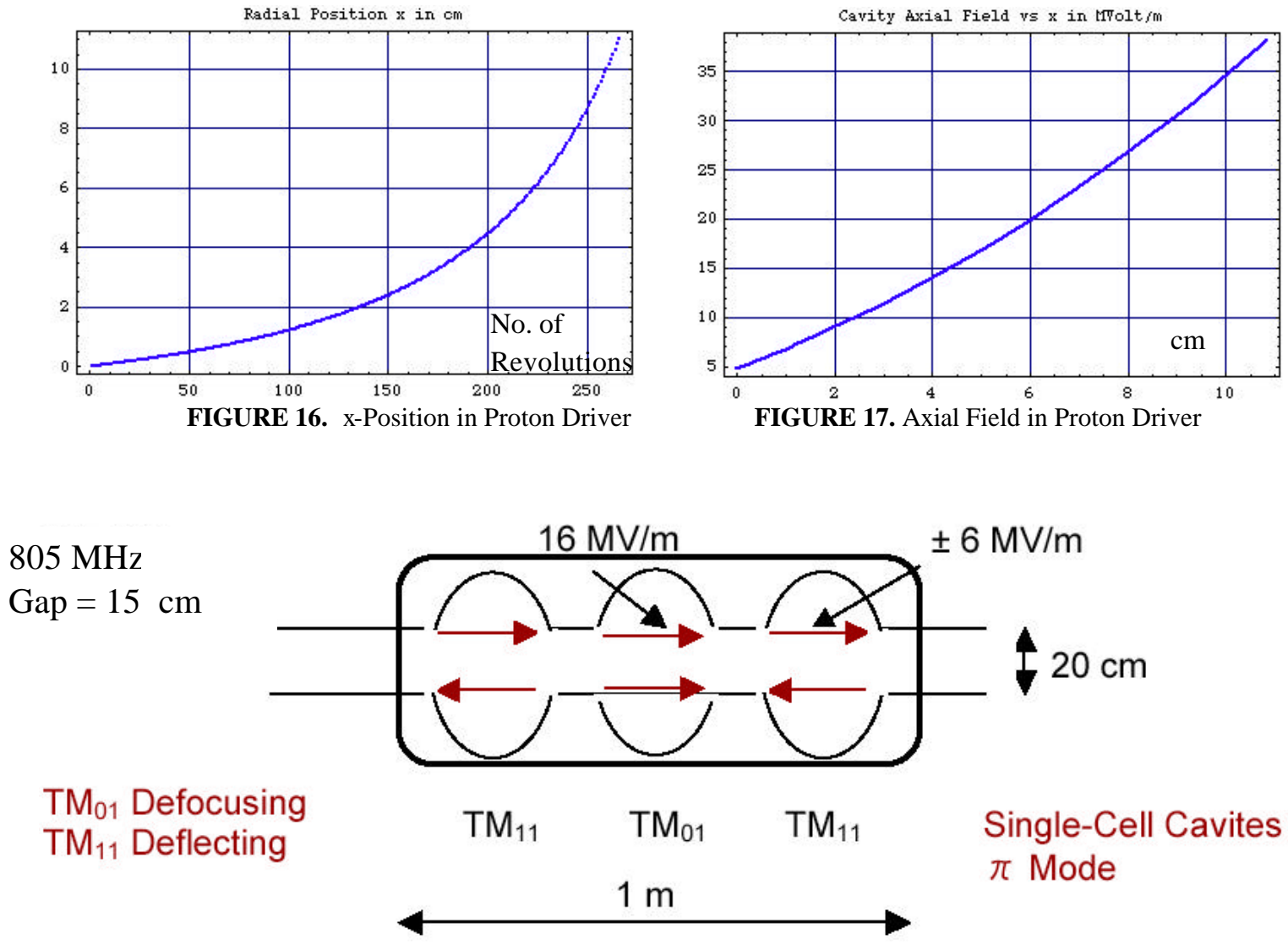

FIGURE 18. Combination of 3 cavities to reproduce behavior of required axial field profile. 


\section{TRACKING}

We performed computer tracking of RF acceleration under different conditions. All the cases reported here apply to the Proton Driver FFAG accelerator.

A. One single cavity with ideal RF voltage as plotted in Figure 19. Constant RF phase of $60^{\circ}$. No betatron oscillations, only longitudinal motion. Tracking of single particles. Initial conditions: kinetic energy of $250 \mathrm{MeV}$; no energy deviation $\varepsilon=w-w_{\text {ref }}=0$; initial phase errors $\Delta \phi$ added on top of synchronous RF phase. The tracking results are displayed in Figures 20 and 21 for different values $\Delta \phi=0,0.01,0.02,0.03$, and 0.05 . Both plots are $\varepsilon$ in $\mathrm{MeV}$ versus $\tau=\Delta \phi / 2 \mathrm{p} f_{\mathrm{RF}}$ in nsec. Figure 22 shows the analytical calculation, after linearization of the equations of motion, of the RF bucket area during acceleration. The value at the top is $0.008 \mathrm{eV}$-s. Tracking shows that the largest amplitude of stable motion is about $\Delta \phi=0.05$ corresponding to about $0.006 \mathrm{eV}$-s showing a good agreement with the analytical expectation.

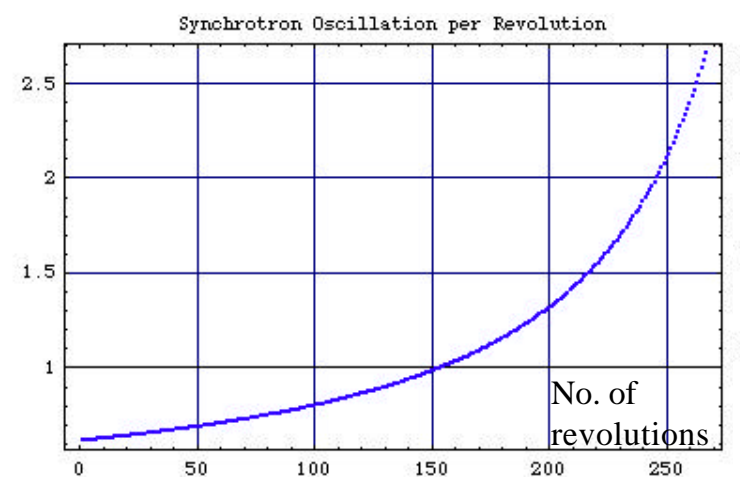

FIGURE 19. RF Peak Voltage in Proton Driver

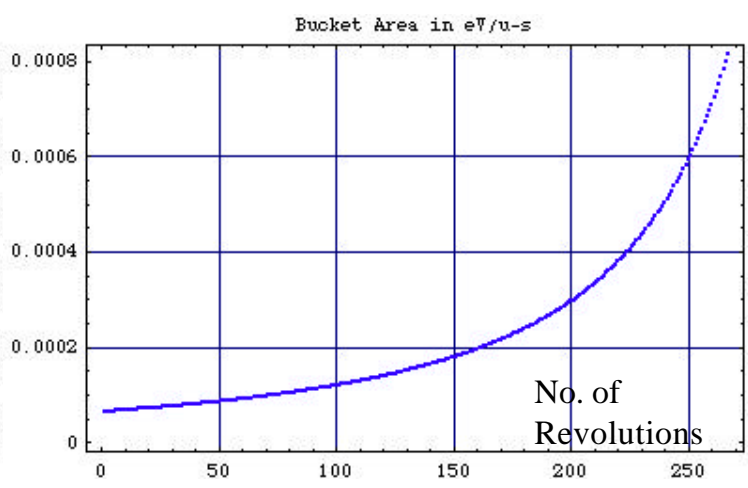

FIGURE 22. Bucket Area in Proton Driver

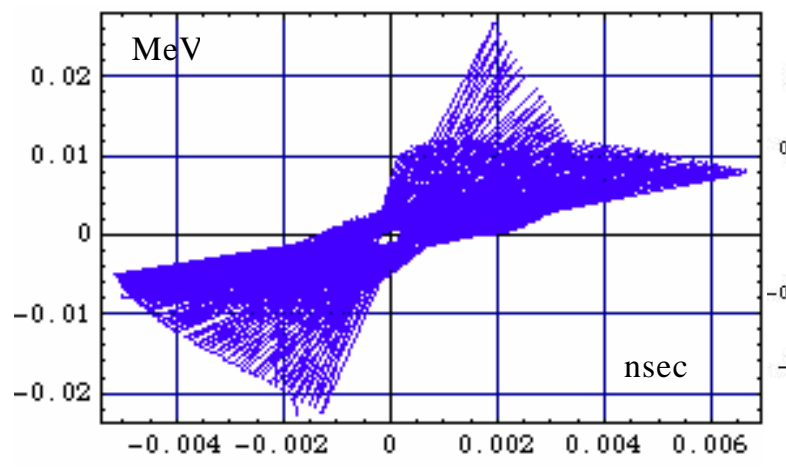

FIGURE 20. Phase Plot $\Delta \phi=0$ and 0.01

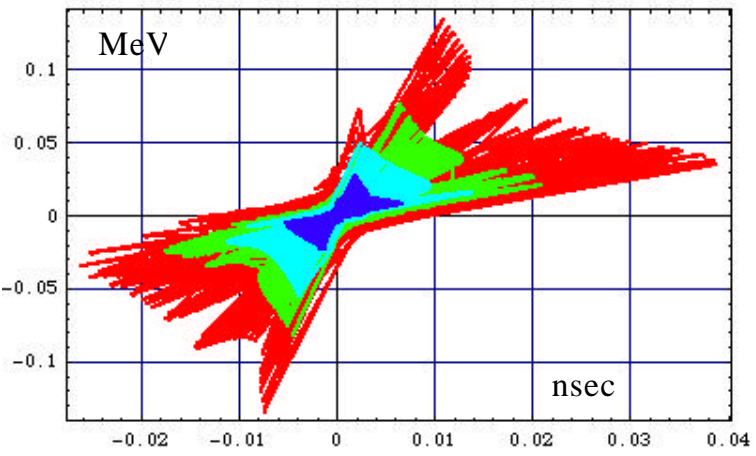

FIGURE 21. $\Delta \phi=0.01,0.02,0.03$ and 0.05

B. The previous tracking results showed an exceedingly large synchrotron oscillation frequency that can be compared with the result of the analytical calculation plotted in Figure 23 that displays number of oscillations per revolution versus number of revolutions during acceleration. We have done then more computer tracking including also betatron oscillations to check for potential instability because of betatron-synchrotron coupling. We have set initially the vertical motion to be identically zero, $\mathrm{y}=\mathrm{y}^{\prime}=0$, and increased the initial radial displacement from $\Delta \mathrm{x}=$ 0 (in Figures 24, 25 and 26) to 10 and $30 \mathrm{~mm}$ (respectively in Figures 27 and 28), always with $\Delta x^{\prime}=0$. The motion seems to be stable also with very large initial amplitude, but on the other end no non-linear errors have been added in the simulation. 


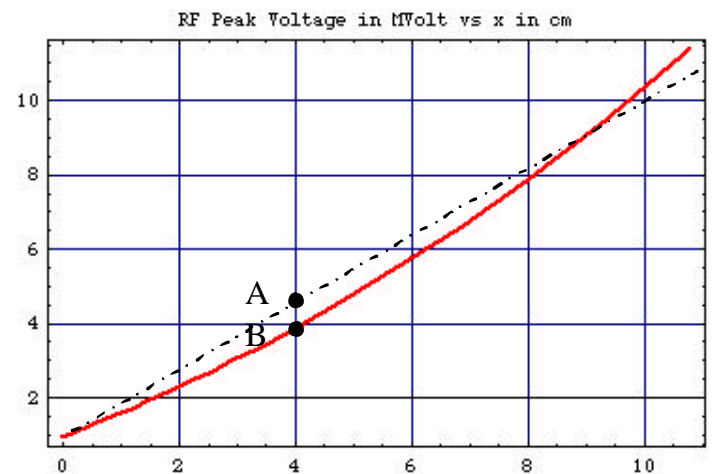

FIGURE 23. RF Peak Voltage in Proton Driver

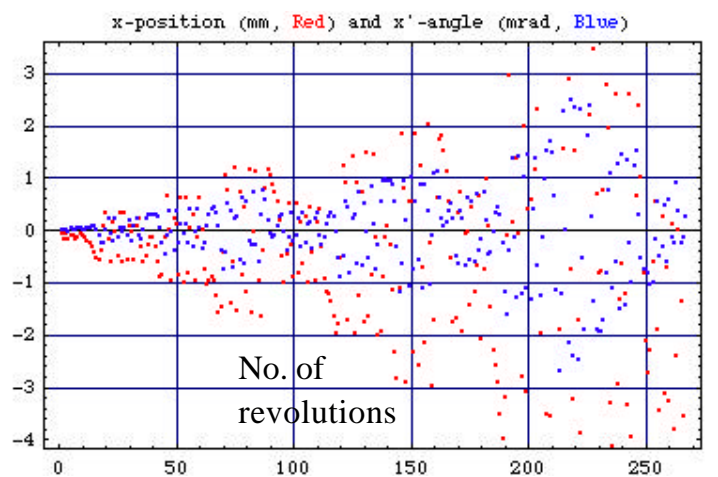

FIGURE 24. $x$ and $x^{\prime}$ during acceleration in PD
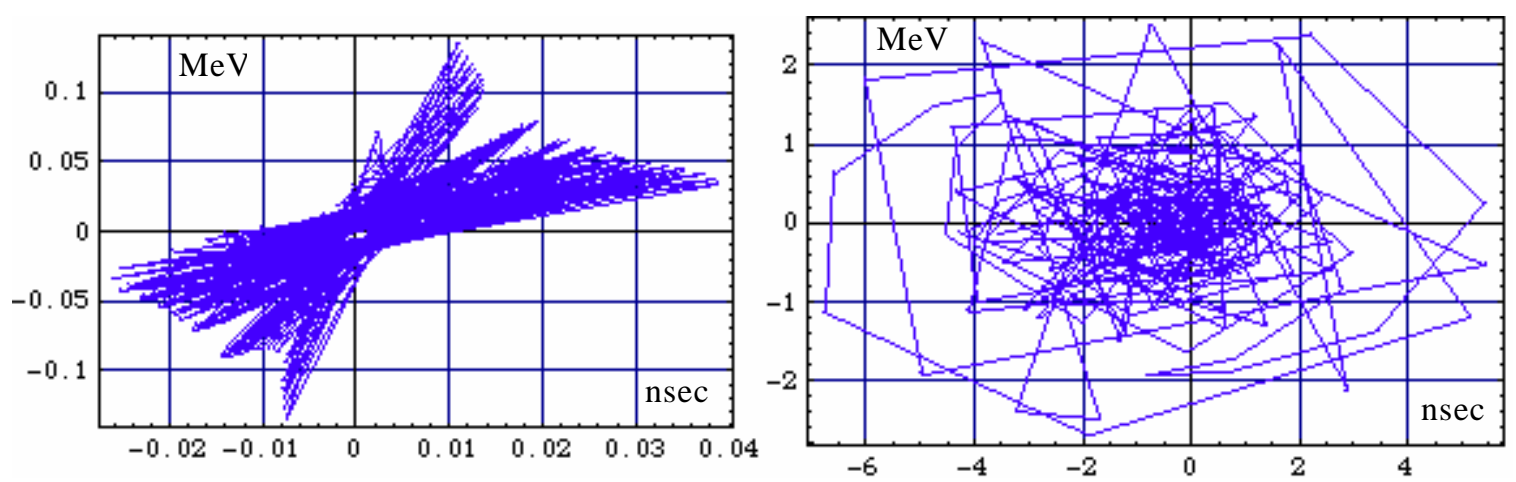

FIGURE 25. Phase Plot for $\Delta \mathrm{x}=0 \mathrm{~mm}$ without $\beta$-oscillations

FIGURE 26. $\Delta \mathrm{x}=0 \mathrm{~mm}$ with $\beta$-oscillations

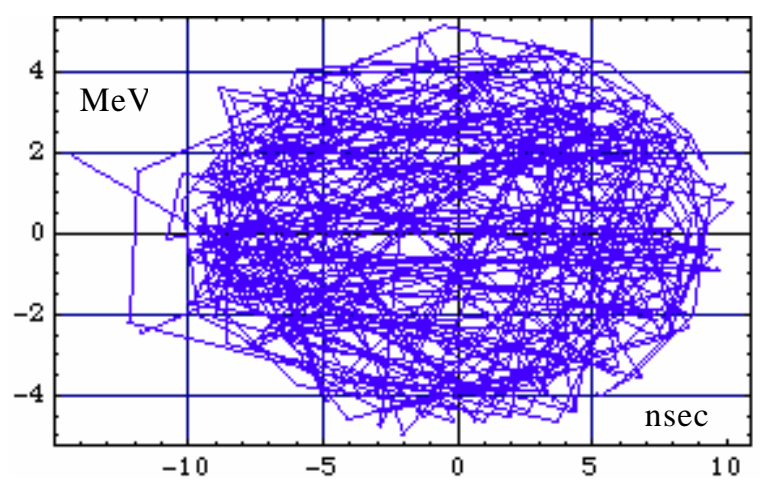

FIGURE 27. $\Delta \mathrm{x}=10 \mathrm{~mm}$ with $\beta$-oscillations

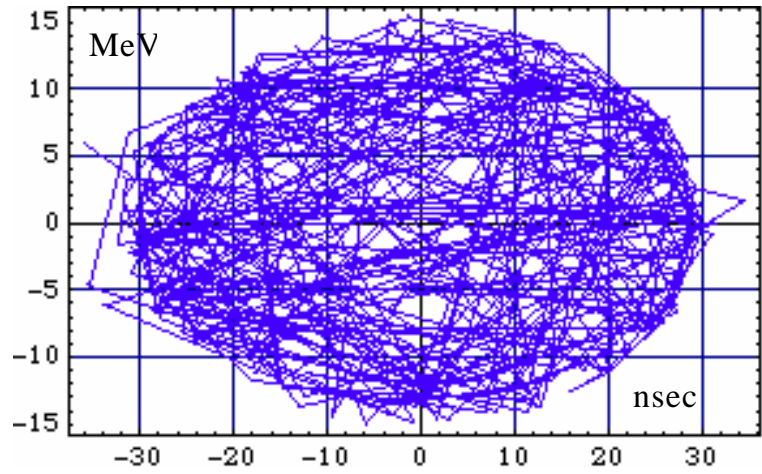

FIGURE 28. $\Delta \mathrm{x}=30 \mathrm{~mm}$ with $\beta$-oscillations

C. Between cavity crossings, every revolution, the particle 4 vector was multiplied by the corresponding 4 x 4 transfer matrix according to

$$
\left|\begin{array}{l}
\mathrm{x} \\
\mathrm{x}^{\prime} \\
\Delta \mathrm{s} \\
\delta
\end{array}\right|_{2}=\left|\begin{array}{llll}
\mathrm{m}_{11} & \mathrm{~m}_{12} & 0 & \mathrm{~m}_{14} \\
\mathrm{~m}_{21} & \mathrm{~m}_{22} & 0 & \mathrm{~m}_{24} \\
\mathrm{~m}_{31} & \mathrm{~m}_{34} & 1 & \mathrm{~m}_{34} \\
0 & 0 & 0 & 1
\end{array}\right| \quad\left|\begin{array}{l}
\mathrm{x} \\
\mathrm{x}^{\prime} \\
\Delta \mathrm{s} \\
\delta
\end{array}\right| 1
$$


The variation of the path length with betatron oscillations if of course also taken into account. We plot the result in Figures 29, 30 and 31 for $\Delta \mathrm{x}=0 \mathrm{~mm}, 10 \mathrm{~mm}$ and $30 \mathrm{~mm}$ respectively. Of course the contribution $\Delta \mathrm{s} / \mathrm{C}$ to $\Delta \mathrm{T} / \mathrm{T}$ from the path length can be neglected when compared to the contribution from the change of the beam velocity shown in Figure 32.

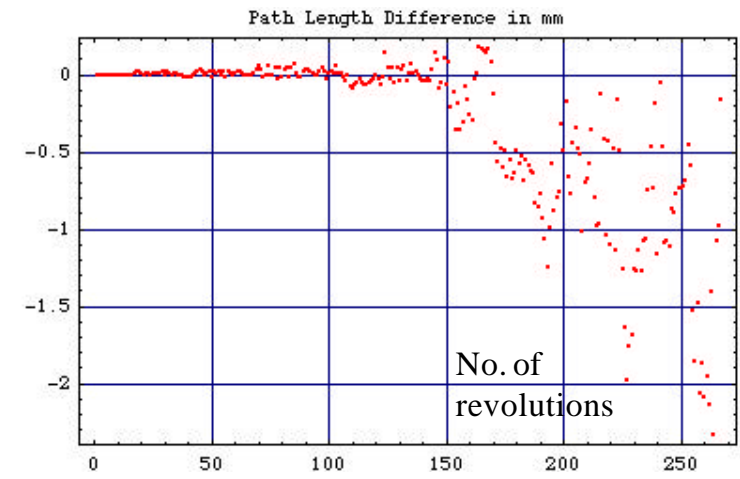

FIGURE 29. Path Length with $\Delta \mathrm{x}=0 \mathrm{~mm}$

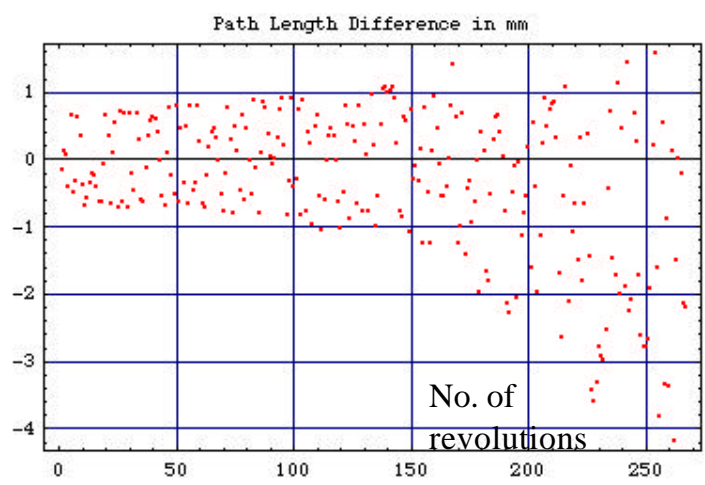

FIGURE 31. Path Length with $\Delta \mathrm{x}=30 \mathrm{~mm}$

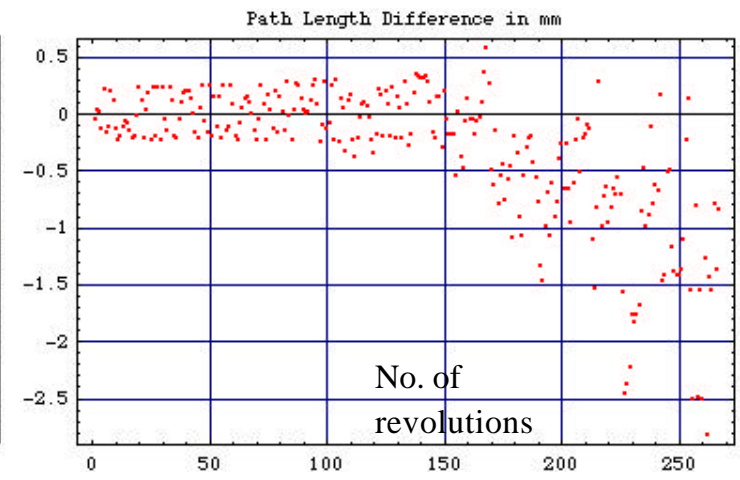

FIGURE 30. Path Length with $\Delta \mathrm{x}=10 \mathrm{~mm}$

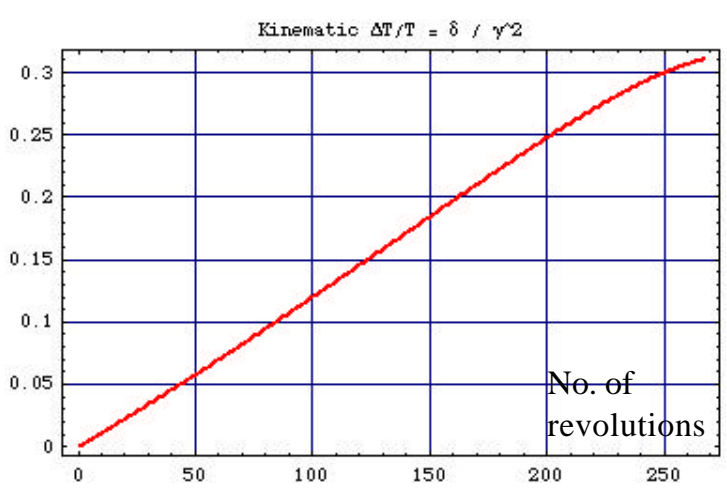

FIGURE 32. Relative Time of Flight

D. We now turn our attention to the case that there are 3 RF cavity systems located next to each other, one period of the FFAG ring apart, as shown in Figure 33. Each cavity group is made of a combination of cavities as that shown in Figure 18. The grouping in multiple cavities is to void exceeding the allowable limit of the cavity axial field, and to generate an energy gain profile as that given by Figure 11. All cavities operate with the same RF voltage $V_{1}$ that of course will vary from turn to turn as the beam moves radially during acceleration. The group of cavities in the center " 1 " operates at the constant RF phase $\phi_{s}=60^{\circ}$. The other two groups " 2 " and " 3 " are respectively advanced and delayed in phase by an angle $\alpha$ that may also vary from turn to turn. Thus the net energy gain per revolution is given by

$\mathrm{V}_{\mathrm{n}} \sin \phi_{\mathrm{s}}=\mathrm{V}_{1}\left[\sin \left(\phi_{\mathrm{s}}-\alpha\right)+\sin \left(\phi_{\mathrm{s}}\right)+\sin \left(\phi_{\mathrm{s}}+\alpha\right)\right]$

The phase difference $\alpha$ is to be adjusted to compensate for the time lag between crossings of the cavities. This condition is called paraphasing since in this mode all cavities together act on the beam as a single one located at the center. As the beam leaves cavity " 1 " it would be ideal if it enters cavity " 2 " with the same phase $\phi_{s}$. In the example of FFAG ring we study here there are $\mathrm{P}=80$ periods each of length $\mathrm{L}=$ $204 \mathrm{~m} / 80=2.55 \mathrm{~m}$ (neglecting the variation with the particle momentum). At the frequency of $805 \mathrm{MHz}$ the RF wavelength $\lambda=0.3723 \mathrm{~m}$. Obviously $\mathrm{L}$ and $\lambda$ are not in an integral relation since $\mathrm{L} / \lambda=7-0.1507$. But it is only the fractional part that

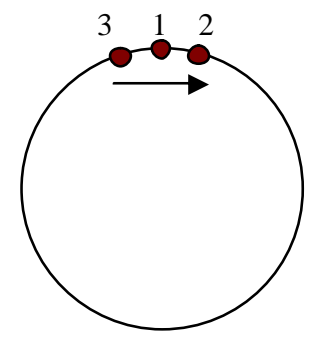

FIGURE 33. FFAG Ring with a group of 3 RF Cavities 


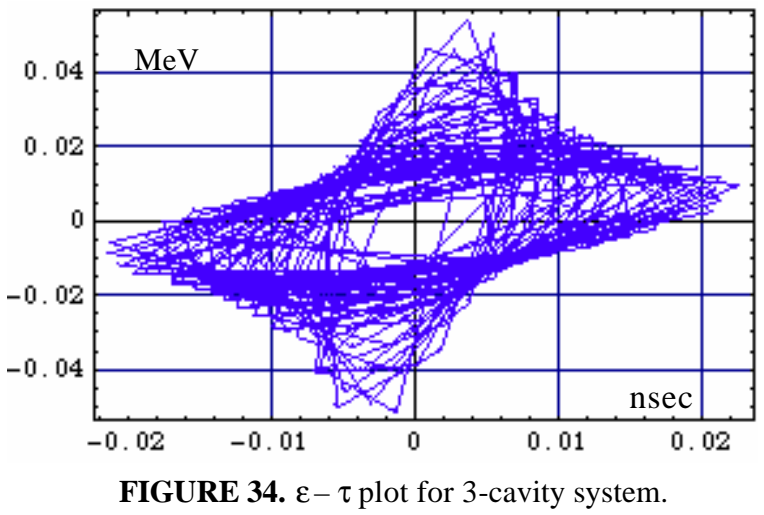

needs to be compensated by the phase difference $\alpha=2 \mathrm{p} f_{\mathrm{RF}}$ $\mathrm{T} / \mathrm{P}$ where $\mathrm{T}$ is the revolution period and $\mathrm{T} / \mathrm{P}$ the time it takes to travel from cavity group " 1 " to cavity group " 2 " (or from cavity group "3" to cavity group " 1 "). We have done numerical tracking for this configuration and these parameters. The results for the entire acceleration cycle are shown in Figure 34. The revolution Period $\mathrm{T}$ of course varies during acceleration, and so it does the phase difference $\alpha$.

\section{MORE WORK TO BE DONE}

The preliminary results of the numerical simulation described in this note are very encouraging for the practical use of the acceleration method by the HNJ. Nevertheless there remain more issues that need to be addressed with further study of the method. We mention here two issues among the most relevant and important, in our opinion.

Issue \# 1. Figure 19 shows the Radial Field Profile of all the cavities combined. It can be approximated with a Linear Profile, also shown in the same Figure by the dashed straight line, probably more technically feasible to realize in practice. A preliminary computer tracking of this linearization procedure though failed. Probably, and this should be checked, the actual profile should be approximated with a sequence of shorter straight lines. The question is how large an error on the energy gain profile can be tolerated without endangering the stability of the beam during acceleration. The question is actually even more general: how close it is required to follow precisely the energy gain profile, considering also the fact that the actual field distribution in a group of cavities as that shown in Figure 18 is likely given by a combination of Bessel functions. For instance, taking up again the example of Figure 19, when the beam is at a certain displacement $\mathrm{x}$ the required RF peak voltage is the one corresponding to point $\mathrm{B}$, whereas the actual value applied corresponds to A. The energy gain difference $\Delta w$ between the two points may be too large and causes the particle to be lost. From inspection of Figure 21 it is seen that $\Delta w$ should be well within $\pm 0.1 \mathrm{MeV}$.

Issue \# 2. The energy gain profile needed for the HNJ method can be achieved with a system of 3 cavities placed together in one spot as shown in Figure 18. The $\mathrm{TM}_{01}$ cavity in the middle has a solenoid magnetic field that alters the focusing of the transverse motion. It can be compensated in principle with the external focusing elements. Of a more serious effect are though the two $\mathrm{TM}_{11}$ cavities on the side, since they introduce a net lateral (radial) deflection of the beam motion. The deflection can be cancelled by two consecutive groups of 3 cavities each placed at $180^{\circ}$ betatron phase advance. In a Scaling FFAG lattice, if such locations are found, then the phase advance between them remains constant during acceleration. But in a Non-Scaling FFAG lattice, usually the required phase advance is obtained only at one value of energy, typically just soon after injection. The phase advance then drops during acceleration and the cancellation of the deflecting modes can only be partial. The effect of this need to be determined with further tracking analysis of the sort explained in this report.

\section{REFERENCES}

1. A.G. Ruggiero, "rf acceleration with harmonic number jump". Physical Review ST-A\&B 9, 100101 (2006).

2. A.G. Ruggiero, "RF Acceleration with Harmonic-Number Jump". BNL Internal Report C-A/AP/\#237, May 2006.

3. A.G. Ruggiero, "RF Acceleration by HJ in FFAG Accelerators". ICFA Beam Dynamics Newsletter No. 43. Editors: C.R. Prior and W. Chou. August 2007. Pages 88-96.

4. A.G. Ruggiero, "FFAG-based Proton and Heavy-Ion High-Power Drivers". ICFA Beam Dynamics Newsletter No. 43. Editors: C.R. Prior and W. Chou. August 2007. Pages 77-87.

5. M. Tanigaki et al., "Status of FFAG complex at KURRI", The Int. Workshop on FFAG Accel., page 1, Dec. 5-9, 2005, KURRI, Osaka, Japan. 